\section{How to book for microarrays}

\author{
DNA Microarrays: A Molecular Cloning Manual \\ Edited by David Bowtell and Joseph Sambrook \\ Cold Spring Harbor Laboratory Press, \$195 (hardcover), ISBN-0-87969-624-9, 2003.
}

\section{Reviewed by Adam Carroll}

University of California-San Francisco, Core Facility for Genomics and Proteomics, San Francisco, California, USA.

As someone who teaches the fabrication and use of DNA microarrays professionally, I took more than a casual interest in Cold Spring Harbor Laboratory Press' recent offering, DNA Microarrays: A Molecular Cloning Manual. I was pleased to find it very useful, in keeping with the tradition of the Molecular Cloning series. This book will help a lot of people make a lot of data a lot better.

One of the most attractive features of the book is its comprehensive coverage of microarray technology. The editors, David Bowtell and Joseph Sambrook, have gathered scores of protocols and copious expertise from many labs that use microarrays. Using such a broad base of informa-

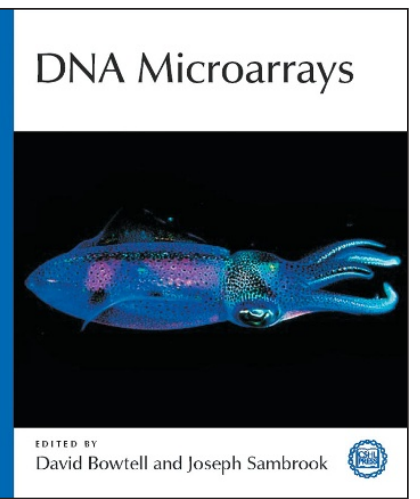

experiments midstream. Those far enough into a project to be experiencing problems will appreciate the troubleshooting sections, which include images of array artifacts and suggested solutions. Readers will find an alternative protocol for almost any step of their experiments, along with a discussion of its advantages and disadvantages. The illustrations of the arrays and how the techniques are done are helpful.

One negative consequence of this breadth of information is that some techniques are described in only enough detail to make sense to someone who already knows what to do. For example, if you don't know how to use a Narishige micromanipulator to do tissue tion will make the book applicable to many kinds of arrays, from commercial oligonucleotide arrays to tissue sample arrays fabricated in-house, and many kinds of samples, from common to rare samples isolated from various organisms.

Such a large amount of information could be asphyxiating, but thankfully, the book is organized in chronological order starting with the printing and preparation of the arrays themselves and ending with the gathering and analysis of the data. In most cases, the book describes several alternatives to any protocol. Similarly, different options for software tools are tabulated in the relevant places throughout the text with information about vendor, source and features.

Because so many different experiments and protocols are described, this book will be particularly useful to researchers who want to vary the conditions of their microarray analyses or carry out related
Because of the relative youth of the microarrays field and the nature of compiling protocols from many authors and labs, alternative protocols for the same procedure often contain different recommendations for instruments or commercial kits, which means that researchers must pay close attention to avoid purchasing redundant supplies and equipment. For example, a multichannel pipettor would be unnecessary for resuspending plates if you have already purchased a 96channel pipetting robot.

Furthermore, because experts in this field are still refining their protocols, the newest and most effective techniques might be found on the Internet (or in the next edition of this book). It will also be interesting to see how many of the commercial vendors listed in this edition of the book will still be selling similar wares the next time around. This might be particularly true for software tools that are being developed rapidly.

My only criticism regarding the book is that the sections covering informatics are at the end. Although this organization fits well with the chronology of an experiment, it means that the most-often neglected or misunderstood, yet crucial, aspect of a microarray experiment ends up in the most-often neglected part of the book. The bioinformatics section itself is good and covers the requisite material on data acquisition, storage and analysis, but the section on experimental design, included therein may not receive due consideration from readers until it is too late. The consequence of neglecting experimental design and other bioinformatics issues up front is an experiment that might be painfully difficult to interpret; this is particularly relevant for molecular biologists making their first serious foray into handling the glut of data produced by an array experiment.

DNA Microarrays would make an excellent addition to the library of any group currently involved in microarray experiments or one considering initiating a microarray program. I don't know if the book will ever make good on the promise of some of the contributing authors that "even the inept can succeed," but it does contain the protocols and expertise to make that possible. 